\title{
COVID-19 transmission risk factors
}

\author{
Alessio Notari ${ }^{1}$ and Giorgio Torrieri ${ }^{2}$ \\ ${ }^{1}$ University of Barcelona \\ ${ }^{2}$ State University of Campinas
}

September 21, 2020

\begin{abstract}
We analyze risk factors correlated with the initial transmission growth rate of the recent COVID-19 pandemic in different countries. The number of cases follows in its early stages an almost exponential expansion; we chose as a starting point in each country the first day $\$ \mathrm{~d}_{\_} \mathrm{i} \$$ with 30 cases and we fitted for 12 days, capturing thus the early exponential growth. We looked then for linear correlations of the exponents $\$ \backslash$ alpha $\$$ with other variables, for a sample of 126 countries. We find a positive correlation, $\{\backslash$ it i.e. faster spread of COVID-19\}, with high confidence level with the following variables, with respective $\$ \mathrm{p} \$$-value: low Temperature $\left(\$ 4 \backslash \operatorname{cdot} 10^{\wedge}\{-7\} \$\right)$, high ratio of old vs. ${ }^{\sim}$ working-age people $\left(\$ 3 \backslash \operatorname{cdot} 10^{\wedge}\{-6\} \$\right)$, life expectancy $\left(\$ 8 \backslash \operatorname{cdot} 10^{\wedge}\{-6\} \$\right)$, number of international tourists $\left(\$ 1 \backslash \operatorname{cdot} 10^{\wedge}\{-5\} \$\right)$, earlier epidemic starting date $\$ \mathrm{~d} \_\mathrm{i} \$\left(\$ 2 \backslash \operatorname{cdot} 10^{\wedge}\{-5\} \$\right)$, high level of physical contact in greeting habits $\left(\$ 6 \backslash \operatorname{cdot} 10^{\wedge}\{-5\} \$\right)$, lung cancer prevalence $\left(\$ 6 \backslash\right.$ cdot $\left.10^{\wedge}\{-5\} \$\right)$, obesity in males $\left(\$ 1 \backslash \operatorname{cdot} 10^{\wedge}\{-4\} \$\right)$, share of population in urban areas $\left(\$ 2 \backslash \operatorname{cdot} 10^{\wedge}\{-4\} \$\right)$, cancer prevalence $\left(\$ 3 \backslash \operatorname{cdot} 10^{\wedge}\{-4\} \$\right)$, alcohol consumption $(\$ 0.0019 \$)$, daily smoking prevalence $(\$ 0.0036 \$)$, UV index $(\$ 0.004 \$$, smaller sample, 73 countries), low Vitamin D serum levels (\$0.002-0.006\$, smaller sample, $\$ \backslash$ sim $50 \$$ countries). There is highly significant correlation also with blood type: positive correlation with types $\mathrm{RH}-\left(\$ 3 \backslash \operatorname{cdot} 10^{\wedge}\{-5\} \$\right)$ and $\mathrm{A}+\left(\$ 3 \backslash \operatorname{cdot} 10^{\wedge}\{-3\} \$\right)$, negative correlation with B+ $\left(\$ 2 \backslash \operatorname{cdot} 10^{\wedge}\{-4\} \$\right)$. We also find positive correlation with moderate confidence level (\$p\$-value of $\left.\$ 0.02 \backslash \operatorname{sim} 0.03 \$\right)$ with: CO $\$ \_-$ $2 \$$ /SO emissions, type- 1 diabetes in children, low vaccination coverage for Tuberculosis (BCG). Several of the above variables are correlated with each other and likely to have common interpretations. We thus performed a Principal Component Analysis, in order to find the significant independent linear combinations of such variables. We also analyzed the possible existence of a bias: countries with low GDP-per capita, typically located in warm regions, might have less intense testing and we discuss correlation with the above variables
\end{abstract}

\section{Hosted file}

NEWpaperCOVID-multigtER-apa.pdf available at https://authorea.com/users/360787/articles/ 482318-covid-19-transmission-risk-factors 